\title{
THE CONSTRUCTION OF ALGEBRAIC CORRESPONDENCES BETWEEN
}

\section{TWO ALGEBRAIC CURVES*}

BY

VIRGIL SNYDER AND F. R. SHARPE

1. Statement of the problem. Given two algebraic curves, $C\left(x_{1}, x_{2}, x_{3}\right)$ $\equiv C(x)=0$ of genus $p$ in the plane $(x)$, and $C^{\prime}\left(x^{\prime}\right)=0$ of genus $p^{\prime}$ in the plane $\left(x^{\prime}\right)$. Suppose that to a point $(y)$ on $C$ correspond $n^{\prime}$ points $\left(y^{\prime}\right)$ on $C^{\prime}$, and that to a point $\left(y^{\prime}\right)$ on $C^{\prime}$ correspond $n$ points $(y)$ on $C$. The two curves $C, C^{\prime}$ are then said to be in $\left(n, n^{\prime}\right)$ correspondence. It is the purpose of this paper to give some methods of constructing curves having such correspondences, and of obtaining the equations which define them.

For certain positions of the point $(y)$, two of the $n^{\prime}$ images on $C^{\prime}$ may coincide. Such a point is called a branch-point, and the image point that is counted twice is called a coincidence. If the number of branch-points on $C$ is denoted by $\eta$, and on $C^{\prime}$ by $\eta^{\prime}$, then we have by Zeuthen's formula

$$
\eta^{\prime}-\eta=n^{\prime}(2 p-2)-n\left(2 p^{\prime}-2\right) .
$$

2. Intermediary curve. Let $C, C^{\prime}$ lie in different planes in ordinary space. Connect each point $(y)$ of $C$ with all the corresponding points $\left(y^{\prime}\right)$ on $C^{\prime}$ by means of straight lines; similarly, connect each point $\left(y^{\prime}\right)$ on $C^{\prime}$ with all its image points on $C$. In this way a ruled surface $R$ is generated, having $C$ for curve of multiplicity $n^{\prime}$ and $C^{\prime}$ of multiplicity $n$. Let $K$ be an arbitrary plane section of $R$, and let $P$ be its genus. Through any point of $K$ passes one and in general only one generator $g$, and this generator meets $C$ in one point. To this point on $C$ correspond $n^{\prime}$ points on $K$, namely, the points in which the $n^{\prime}$ generators through the given point on $C$ meet the curve $K$. Moreover, all the $n^{\prime}$ points on $K$ have just this one image on $C$. The curves $C, K$ are therefore in $\left(1, n^{\prime}\right)$ correspondence. Similarly, $C^{\prime}, K$ are in $(1, n)$ correspondence. Hence $K$ has two involutions, one of order $n$,-genus $p^{\prime}$, the other of order $n^{\prime}$, genus $p$.

A branch-point on $C$ gives rise to a branch-point on $K$, but since to a point on $K$ corresponds only one point on $C$, there can be no coincidences. By

\footnotetext{
* Presented to the Society, September 6, 1920.
} 
applying Zeuthen's formula we therefore have

$$
2 P-2=n^{\prime}(2 p-2)+\eta=n\left(2 p^{\prime}-2\right)+\eta^{\prime} .
$$

We may therefore state the following known

Theorem: Associated with every $\left(n, n^{\prime}\right)$ correspondence between two curves of genera $p, p^{\prime}$ is an intermediary curve of genus $P$, on which exist two involutions, one of order $n^{\prime}$, genus $p$, and another of order $n$, genus $p^{\prime}$.

3. Series contained in a linear series. It has been found by Castelnuovo* that the maximum value of $\eta$ is $2 n\left(n^{\prime}+p^{\prime}-1\right)$ and of $\eta^{\prime}$ is $2 n^{\prime}(n+p-1)$ and that the maximum values are attained simultaneously. In this case the genus $P$ of $K$ has its maximum value. It was also shown that the necessary and sufficient condition that the $\left(n, n^{\prime}\right)$ correspondence between the given curves can be expressed by means of one auxiliary equation is that $\eta$ or $\eta^{\prime}$ attains its maximum value. Let this equation be $\phi\left(x, x^{\prime}\right)=0$.

When $\left(x^{\prime}\right)$ is fixed on $C^{\prime}, \phi\left(x, x^{\prime}\right)=0$ defines a curve in the plane $(x)$ which meets $C$ in $n$ points, images of the given point on $C^{\prime}$. Similarly, when $(x)$ is fixed on $C$, the curve $\phi=0$ meets $C^{\prime}$ in $n^{\prime}$ points.

In the older literature no other forms of correspondence were known than this Cayley-Brill theory of correspondence, which is a generalization of the correspondence between two straight lines, as developed by Chasles. $\dagger$ It was pointed out by Hurwitz $\ddagger$ that not all correspondences can be expressed by means of one equation but that every correspondence can be expressed by means of at most two auxiliary equations

$$
\phi_{1}\left(x, x^{\prime}\right)=0, \quad \phi_{2}\left(x, x^{\prime}\right)=0 .
$$

No illustrations are given, nor any properties discussed of correspondences requiring two equations for their definition; such correspondences are called singular correspondences. The two equations (1) define a multiple correspondence between two planes, having the restricted property that the entire image of $C$ is $C^{\prime}$ taken multiply, and similarly all the images of points on $C^{\prime}$ lie on $C$. Our problem is thus equivalent to that of finding such correspondences.

4. Intersection of two ruled surfaces. An example of a curve $K$ having two involutions was given by Amodeo§ and cited by Castelnuovo, $\|$ namely,

* Sulle serie algebriche di grippi di punti appartenenti ad una curva algebrica, $\mathrm{R}$ e $\mathrm{n} \mathrm{d}$. d. R . A c c a d e mi a de i Lin ce i, ser. 5, vol. 15(1) (1906), pp. 337-344.

† See Clebsch-Lindemann, Vorlesungen ueber Geometrie, vol. 1, p. $437 \mathrm{ff}$.

$\ddagger$ Ueber algebraische Correspondenzen und das verallgemeinerte Correspondenzprincip, $\mathbf{M}$ a $\mathrm{t} \mathrm{h}$ e matis che Annalen, vol. 28 (1887), pp. 561-593.

$\S \mathrm{F}$. Amodeo, Contribuzione alla teoria delle serie irrazionale involutorie giacenti sulle varieta algebriche ad una dimensione, A n $\mathrm{n}$ a $\mathrm{i}$ di Mat e matica, ser. 2, vol. 20 (1892), pp. 229-235.

\| L. c., p. 342. 
the curve of intersection of two ruled surfaces in general position. Noether* had proved that the intersection $K$ of $R_{m}$, of genus $\pi$ with $R_{m^{\prime}}$, of genus $\pi^{\prime}$ has the genus $P$ defined by

$$
P=(m-1)\left(m^{\prime}-1\right)+m \pi+m^{\prime} \pi^{\prime} .
$$

Since $m, \pi$ are precisely the order and genus of one involution on $K$, and $m^{\prime}, \pi^{\prime}$ are the order and genus of the other, it follows from Castelnuovo's theorem that $P$ has its maximum value and therefore that the correspondence can be expressed by one equation. Analytically the equations of a generator of $R$ may be written in the form $\Sigma a_{i} x_{i}=0, \Sigma b_{i} x_{i}=0$ where $a_{i}$ and $b_{i}$ are rational functions of parameters $\lambda_{1}, \lambda_{2}, \lambda_{3}$ connected by an algebraic relation $f\left(\lambda_{1}, \lambda_{2}, \lambda_{3}\right)=0$ of genus $\pi$. Similarly, for $R^{\prime}$ we have $\Sigma c_{i} x_{i}=0$, $\Sigma d_{i} x_{i}=0$, where $c_{i}, d_{i}$ are functions of $\left(\lambda^{\prime}\right)$, and $f^{\prime}\left(\lambda^{\prime}\right)=0$.

The condition that the generators intersect is

$$
\Delta \equiv\left(a_{1}, b_{2}, c_{3}, d_{4}\right)=0 .
$$

This equation expresses the correspondence between

$$
f\left(\lambda_{1}, \lambda_{2}, \lambda_{3}\right)=0 \quad \text { and } \quad f^{\prime}\left(\lambda_{1}^{\prime}, \lambda_{1}^{\prime}, \lambda_{3}^{\prime}\right)=0 .
$$

5. Ruled surfaces with common generators. For certain sets of values of $(\lambda)$ and of $\left(\lambda^{\prime}\right)$ it may happen that the four planes $(a),(b),(c)$, and $(d)$ have a line in common, instead of simply a point of $K$. Such a line is a common generator of $R$ and $R^{\prime}$. For each common generator it can be proved that the genus of $K$ is reduced by unity, but since each common generator counts for two coincidences, Castelnuovo's condition is still satisfied, and one equation is sufficient to determine the correspondence.

6. Ruled surfaces with common plane section $\bar{C}$. Since any plane section of $R$ or of $R^{\prime}$ is in $(1,1)$ correspondence with $\bar{C}$, the correspondence is equivalent to a correspondence on $\bar{C}$. A generator of $R$ through a point $(y)^{\text {a }}$ of $\bar{C}$ meets the residual curve $K$ in points through each of which passes a generator of $R^{\prime}$, and this generator meets $\bar{C}$ in an image point $\left(y^{\prime}\right)$. We have thus a correspondence of valence 1 .

Any case of this kind is an example of the type

$$
\begin{gathered}
f\left(x_{1}, x_{2}, x_{3}\right)=0, \\
f\left(x_{1}^{\prime}, x_{2}^{\prime}, x_{3}^{\prime}\right)=0, \\
A\left(x_{1} x_{3}^{\prime}-x_{1}^{\prime} x_{3}\right)+B\left(x_{2} x_{3}^{\prime}-x_{2}^{\prime} x_{3}\right)=0 .
\end{gathered}
$$

If between (2) and (4) we eliminate $x_{1}$, then by means of (3) the factor $x_{2} x_{3}^{\prime}$

* M. Noether, Zur Theorie des eindeutigen Entsprechens algebraischer Gebilde, M a t h e mat is che Annalen, vol. 8 (1875), pp. 495-533.

Trans. Am. Math. Soc. 3 
$-x_{2}^{\prime} x_{3}$ can be removed; we thus obtain a fourth relation, which completes the definition of the correspondence.

Example: Thus, the two ruled surfaces

$$
\begin{aligned}
R & \equiv x_{2}^{2} x_{3}\left(x_{1}+x_{4}\right)=x_{1}^{2}\left(x_{1}^{2}+2 x_{1} x_{4}+x_{4}^{2}+x_{3}^{2}\right), \\
R^{\prime} & \equiv x_{1} x_{2}^{2} x_{3}=\left(x_{1}+x_{4}\right)^{2}\left(x_{1}^{2}+x_{3}^{2}\right)
\end{aligned}
$$

pass through the curve $f \equiv x_{2}^{2} x_{3}-x_{2}\left(x_{1}^{2}+x_{3}^{2}\right)=0, x_{4}=0$. The residual curve of intersection of $R$ and $R^{\prime}$ defines the $(2,2)$ correspondence on $f$ expressed by

$$
x_{1}^{\prime 2} x_{2} x_{3}-x_{1}^{2} x_{2}^{\prime} x_{3}^{\prime}=0, \quad x_{2} x_{3} x_{1}^{\prime 2}+x_{1} x_{3} x_{1}^{\prime} x_{3}^{\prime}+x_{1} x_{2} x_{3}^{\prime 2}=0 .
$$

7. Ruled surfaces with common plane multiple curve $\bar{C}$. Since any plane sections $C, C^{\prime}$ are in $(k, 1),\left(k^{\prime}, 1\right)$ correspondence with $\bar{C}$ respectively, the equations of $C$ and of $C^{\prime}$ may be written in the forms $f\left(\phi_{1}, \phi_{2}, \phi_{3}\right)=0$, $f\left(\phi_{1}^{\prime}, \phi_{2}^{\prime}, \phi_{3}^{\prime}\right)=0$, where the equations

$$
y_{i}=\phi_{i}\left(x_{1}, x_{2}, x_{3}\right)=0, \quad y_{i}^{\prime}=\phi_{i}^{\prime}\left(x_{1}^{\prime}, x_{2}^{\prime}, x_{3}^{\prime}\right)
$$

define the $(k, 1),\left(k^{\prime}, 1\right)$ correspondences respectively. Any cases of this type can now be expressed by

$$
f\left(\phi_{1}, \phi_{2}, \phi_{3}\right)=0, \quad f\left(\phi_{1}^{\prime}, \phi_{2}^{\prime}, \phi_{3}^{\prime}\right)=0, \quad\left|\begin{array}{lll}
A & B & C \\
\phi_{1} & \phi_{2} & \phi_{3} \\
\phi_{1}^{\prime} & \phi_{2}^{\prime} & \phi_{3}^{\prime}
\end{array}\right|=0
$$

To obtain a fourth equation the procedure is exactly the same as in the preceding example.

8. Ruled surfaces whose generators are multiple secants of a common curve $\bar{C}$. A curve $\bar{C}$ can be found on $R$ having the generators of $R$ for multiple secants of any order. Another ruled surface $R^{\prime}$ can be constructed having for generators the bisecants of $\bar{C}$ which meet a given curve; still another may be found by the trisecants of $\bar{C}$.

Example: The bisecants of a space quartic $\bar{C}$ of genus 1 which meet a fixed line lie on a ruled surface $R$ of order 8 , having the line double, and $\bar{C}$ to multiplicity three. Let $\bar{C}$ be the intersection of the quadrics $\sum x_{i}^{2}=0 \sum a_{i} x_{i}^{2}=0$ and the line be $x_{1}=0, x_{2}=0$. This line passes through two of the vertices of the self-polar tetrahedron with respect to the pencil of quadrics through $\bar{C}$. The ruled surface is composite, consisting of a quartic $R$ and of the two quadric cones with vertices $(0,0,0,1),(0,0,1,0)$ and passing through $\bar{C}$. The two generators of $R$ through a point $\left(0,0, y_{3}, y_{4}\right)$ are the intersection of the quadric of the pencil through the point

$$
\begin{aligned}
\left(y_{3}^{2}+y_{4}^{2}\right)\left(a_{1} x_{1}^{2}+a_{2} x_{2}^{2}\right)-\left(a_{3} y_{3}^{2}+a_{4} y_{4}^{2}\right)\left(x_{1}^{2}+x_{2}^{2}\right) & \\
& +\left(a_{3}-a_{4}\right)\left(y_{4}^{2} x_{3}^{2}-y_{3}^{2} x_{4}^{2}\right)=0
\end{aligned}
$$


with the tangent plane to the quadric at this point, thus

The equation of $R$ is

$$
y_{4} x_{3}-y_{3} x_{4}=0 \text {. }
$$

(5) $\quad R \equiv\left(x_{3}^{2}+x_{4}^{2}\right)\left(a_{1} x_{1}^{2}+a_{2} x_{2}^{2}\right)-\left(a_{3} x_{3}^{2}+a_{4} x_{4}^{2}\right)\left(x_{1}^{2}+x_{2}^{2}\right)=0$.

Similarly, the equation of a ruled surface $R^{\prime}$ whose generators are the bisecants of $\bar{C}$ which meet $x_{1}=0, x_{3}=0$ is

$$
R^{\prime} \equiv\left(x_{2}^{2}+x_{4}^{2}\right)\left(a_{1} x_{1}^{2}+a_{3} x_{3}^{2}\right)-\left(a_{2} x_{2}^{2}+a_{4} x_{4}^{2}\right)\left(x_{1}^{2}+x_{3}^{2}\right)=0 .
$$

For $C$ and $C^{\prime}$ we take the sections of these surfaces by the plane $x_{2}=x_{3}$. Their equations are

$$
\begin{gathered}
C \equiv\left(x_{2}^{2}+x_{4}^{2}\right)\left(a_{1} x_{1}^{2}+a_{2} x_{2}^{2}\right)-\left(x_{1}^{2}+x_{2}^{2}\right)\left(a_{3} x_{2}^{2}+a_{4} x_{4}^{2}\right)=0 \\
C^{\prime} \equiv\left(x_{2}^{\prime 2}+x_{4}^{\prime 2}\right)\left(a_{1} x_{1}^{\prime 2}+a_{3} x_{2}^{\prime 2}\right)-\left(x_{1}^{\prime 2}+x_{2}^{\prime 2}\right)\left(a_{2} x_{2}^{\prime 2}+a_{4} x_{4}^{\prime 2}\right)=0
\end{gathered}
$$

We shall now use $y_{i}$ for current coördinates. The generator of $R$ through $(x)$ is given by

$$
x_{2} y_{1}=x_{1} y_{2}, \quad x_{4} y_{3}=x_{2} y_{4},
$$

and the generator of $R^{\prime}$ through $\left(x^{\prime}\right)$ by

$$
x_{2}^{\prime} y_{1}=x_{1}^{\prime} y_{3}, \quad x_{4}^{\prime} y_{2}=x_{2}^{\prime} y_{4} .
$$

The condition that these two generators intersect is

$$
\Delta \equiv x_{2}^{2} x_{1}^{\prime} x_{4}^{\prime}-x_{2}^{\prime 2} x_{1} x_{4}=0 .
$$

This relation, however, is not sufficient to determine the correspondence. Two of the intersections of (9) and (6) lie on $\bar{C}$ and two on $K$. The two intersections on $C$ lie on the two quadrics and are therefore given by

Hence (11) and

$$
-\frac{y_{2}^{2}}{y_{3}^{2}}=\frac{x_{2}^{2}+x_{4}^{2}}{x_{1}^{2}+x_{2}^{2}}=\frac{a_{3} x_{2}^{2}+a_{4} x_{4}^{2}}{a_{1} x_{1}^{2}+a_{2} x_{2}^{2}}
$$

determine the correspondence associated with $\bar{C}$.

The four intersections of (9) and (6) are given by $\left(x_{2}^{2} y_{2}^{2}+x_{4}^{2} y_{3}^{2}\right)\left(a_{1} x_{1}^{2} y_{2}^{2}+a_{3} x_{2}^{2} y_{3}^{2}\right)-\left(x_{1}^{2} y_{2}^{2}+x_{2}^{2} y_{3}^{2}\right)\left(a_{2} x_{2}^{2} y_{2}^{2}+a_{4} x_{4}^{2} y_{3}^{2}\right)=0$; hence the two intersections on $K$ are given by

Hence (11) and

$$
-\frac{y_{2}^{2}}{y_{3}^{2}}=\frac{\left(a_{3}-a_{4}\right) x_{4}^{2}\left(x_{1}^{2}+x_{2}^{2}\right)}{\left(a_{1}-a_{2}\right) x_{1}^{2}\left(x_{2}^{2}+x_{4}^{2}\right)} .
$$

$$
x_{2}^{2} x_{1}^{\prime 2}\left(a_{1}-a_{2}\right) x_{1}^{2}\left(x_{2}^{2}+x_{4}^{2}\right)=x_{1}^{2} x_{2}^{\prime 2}\left(a_{3}-a_{4}\right) x_{4}^{2}\left(x_{1}^{2}+x_{2}^{2}\right)
$$

uetermine the correspondence associated with $K$. 
9. Cases of $(2,2)$ correspondences. If the curve $K$ has two $(1,2)$ involutions, there is a $(1,1)$ transformation associated with each. The product of these two transformations must be of finite order if $P$ is greater than 1 . A repetition of the correspondence from curve to curve can therefore give only a finite number of images on each curve.

CASE of $P=1$. An elliptic curve with periods $2 \omega, 2 \omega^{\prime}$, has three irrational involutions of order $\dot{2}$ which transform $K$ into elliptic curves $C_{1}, C_{2}, C_{3}$ of periods $\left(\omega, 2 \omega^{\prime}\right),\left(2 \omega, \omega^{\prime}\right),\left(2 \omega, \omega+\omega^{\prime}\right) .^{*}$

Between any two of these curves $C$ a $(2,2)$ correspondence exists, not definable by one equation. A repetition of the process, however, reduces all these curves back to $K$, so that the $(2,2)$ correspondences are each compounded of two $(1,2)$ involutions. Using non-homogeneous coördinates, each involution may be expressed by means of a $(1,2)$ correspondence between the planes $\left(x^{\prime}, y^{\prime}\right)$ and $(x, y)$, where $K$ is a cubic of the form

$$
y^{2}=4\left(x-e_{1}\right)\left(x-e_{2}\right)\left(x-e_{3}\right) \text {. }
$$

If we now put $x=\wp(u), y=\wp^{\prime}(u)$, then the relation between $K(x)$ and $C_{1}\left(x^{\prime}\right)$ is expressed by the equations

$$
x^{\prime}=x+\frac{\left(e_{2}-e_{1}\right)\left(e_{3}-e_{1}\right)}{x-e_{i}}, \quad y^{\prime}=y\left[1-\frac{\left(e_{2}-e_{1}\right)\left(e_{3}-e_{1}\right)}{\left(x-e_{1}\right)^{2}}\right] ;
$$

similar forms exist for $C_{2}$ and $C_{3}$.

If we indicate by $K(u)$ the point on $K$ having the parameter $u$, we may say: given a point $K(u)$, there exists one image point $C_{i}(u)$ on each curve $C_{i}$. The residual image of $C_{1}(u)$ is $K(u+\omega)$, of $C_{2}(u) K\left(u+\omega^{\prime}\right)$, of $C_{3}(u)$ $K\left(u+\omega+\omega^{\prime}\right)$. These four points on $K$ form a closed set. They all have the point $K(-2 u)$ for first tangential.

Case of $P=3$. The quartic $K$ of genus 3

$$
a\left(x_{1}^{4}+x_{2}^{4}\right)+b x_{1}^{2} x_{2}^{2}+c x_{1} x_{2} x_{3}^{2}+d x_{3}^{4}=0
$$

possesses the two $(1,2)$ involutions

$$
\begin{array}{lll}
x_{1}^{\prime}=\left(x_{1}+x_{2}\right) x_{3}, & x_{2}^{\prime}=x_{1} x_{2}, & x_{3}^{\prime}=x_{3}^{2}, \\
x_{1}^{\prime \prime}=\left(i x_{1}+i^{3} x_{2}\right) x_{3}, & x_{2}^{\prime \prime}=x_{1} x_{2}, & x_{3}^{\prime \prime}=x_{3}^{2}
\end{array}
$$

which transform $K$ into elliptic quartics $C_{1}, C_{2}$. The curve $K$ is invariant under the corresponding $(1,1)$ transformations

and.

$$
x_{1}^{\prime}=x_{2}, \quad x_{2}^{\prime}=x_{1}, \quad x_{3}^{\prime}=x_{3}
$$

$$
x_{1}^{\prime}=i x_{1}, \quad x_{2}^{\prime}=i^{3} x_{2}, \quad x_{3}^{\prime}=x_{3} \text { ! }
$$

The product of these two transformations is of period 2

\footnotetext{
* Bianchi, Lezioni sulla teoria delle funzioni ellittiche, Seconda edizione. See p. 485.
} 
The $(2,2)$ correspondence between $C_{1}\left(x^{\prime}\right)$ and $C_{2}\left(x^{\prime \prime}\right)$ is defined by the two equations

$$
4 x_{2}^{\prime} x_{3}^{\prime} x_{2}^{\prime \prime 2}=x_{1}^{\prime 2} x_{2}^{\prime \prime 2}+x_{1}^{\prime \prime 2} x_{2}^{\prime 2}, \quad x_{2}^{\prime} x_{3}^{\prime \prime}=x_{2}^{\prime \prime} x_{3}^{\prime} .
$$

A GENERAL CASE. This case can be generalized by taking for $K$ an equation of the form of a polynomial in $x_{1}^{n}+x_{2}^{n}, x_{1} x_{2}, x_{3}$ equated to zero, and replacing $i$ by $\theta$ where $\theta^{n}=1$. We thus find $2 n$ points on $K$ and $n$ points on each curve $C$ which form closed sets. The equations of the $(2,2)$ correspondence between $C(x)$ and $C^{\prime}\left(x^{\prime}\right)$ are

$$
\begin{gathered}
x_{1}^{2} x_{3}^{\prime 2}-x_{1} x_{1}^{\prime} x_{3} x_{3}^{\prime}\left(\theta+\theta^{-1}\right)+x_{1}^{\prime 2} x_{3}+x_{2} x_{3} x_{3}^{\prime 2}\left(\theta-\theta^{-1}\right)^{2}=0, \\
x_{2} x_{3}^{\prime}-x_{3} x_{2}^{\prime}=0 .
\end{gathered}
$$

The point $(1,0,0)$ is not on $C$ nor on $C^{\prime}$. The genus of $K$ is $(n-1)(n-2) / 2$; hence the $(2,2)$ correspondence is closed. The product of the two linear transformations which leave $K$ invariant is of order 2; the group, generated by them is dihedral of order $2 n$.

10. General case. Analytical form of $K$. Since $K$ possesses two involutions, one of order $n$, the other of order $n^{\prime}$, it follows that if the equations of $C$ and $C^{\prime}$ are $f(x)=0, f^{\prime}\left(x^{\prime}\right)=0$, the equation of $K$ may be written in the two forms $f(\phi(y))=0, f^{\prime}\left(\phi^{\prime}\left(y^{\prime}\right)\right)=0$, where $x_{i}=\phi_{i}(y), x_{1}^{\prime}=\phi_{1}^{\prime}\left(y^{\prime}\right)$ define $\mathrm{a}(1, n)$ and $\left(1, n^{\prime}\right)$ transformation respectively. If no restrictions are put on the coefficients in $f$ and $\phi_{i}$ it is impossible to write $K$ in the form $f^{\prime}\left(\phi^{\prime}\left(y^{\prime}\right)\right)=0$. We may, however seek to find the restrictions on $f$ and $\phi$ so that the second form is possible.

Example: If $f(x)=0$ is a general cubic and each $\phi$ is a general quadratic in $(y)$ we proceed to determine restrictions on the coefficients in $f$ and each $\phi$ so that we may write $K$ in the form

$$
\left(y_{1}^{3}+y_{1}^{2} f_{1}+y_{1} f_{2}+f_{3}\right)^{2}=f_{6},
$$

where $f_{i}$ is a binary form of order $i$ in $y_{2}$ and $y_{3}$. By linear transformations on $(x)$ and $(y)$ we may reduce $f_{1}$ to zero and take

$$
\begin{aligned}
& x_{1}=\phi_{1}=y_{1}^{2}+a y_{2}^{2}+c y_{3}^{2}, \\
& x_{2}=\phi_{2}=y_{1}\left(y_{2}+y_{3}\right)+d y_{2}^{2}+e y_{3}^{2}, \\
& x_{3}=\phi_{3}=y_{2} y_{3} .
\end{aligned}
$$

The form of $f$ is then

$$
\begin{aligned}
x_{1}^{3}+x_{1}^{2} x_{3}+x_{1}\left(A x_{2}^{2}+B x_{2} x_{3}\right. & \left.+C x_{3}^{2}\right) \\
& +D^{3} x_{2}^{3}+E x_{2}^{2} x_{31}+F x_{2} x_{3}^{2}+G x_{3}^{3}=0 .
\end{aligned}
$$

The restrictions on the coefficients are much simplified by taking $A=0$, 
but this is not necessary. If we substitute $x_{i}=\phi_{i}$ from (15) in (16) and make the result identical with (14) we find the solution

$$
\begin{gathered}
a=c=2 D, \quad d=e=-D, \\
A=0, \quad B=\frac{D}{2}-6 D^{3}, \quad C=\frac{1}{4}+D_{\vdots}, \quad E-\frac{D^{2}}{2}, \quad F=\frac{D}{4}-2 D^{3}, \\
f_{2}=3 D^{2}\left(y_{2}^{2}+y_{3}^{2}\right)+\frac{y_{2} y_{3}}{2}, \quad f_{3}=\frac{B}{2} y_{2} y_{3}\left(y_{2}+y_{3}\right)+\frac{D^{3}}{2}\left(y_{2}+y_{3}\right)^{3} .
\end{gathered}
$$

The value of $G$ is arbitrary; the form of $f_{6}$ follows from the preceding values of the other quantities involved. The transformation

$$
\begin{aligned}
& x_{1}^{\prime}=y_{1}^{3}+y_{1} f_{2}+f_{3}, \\
& x_{2}^{\prime}=y_{2} y_{3}^{2}, \\
& x_{3}^{\prime}=y_{3}^{3}
\end{aligned}
$$

reduces $K$ to the sextic of genus 2

$$
x_{1}^{\prime 2} x_{3}^{\prime 3}=f_{6}\left(x_{2}^{\prime}, x_{3}^{\prime}\right) .
$$

Between the curves (16) and (18) exists a $(3,4)$ correspondence defined by the $(1,3)$ correspondence $(17)$ and the $(1,4)$ correspondence defined by (15). If now we eliminate $(y)$ between (15) and (17) we obtain the two equations of the correspondence.

11. Surfaces defined by pairs of points of two algebraic curves. An important method of representing multiple correspondences between two curves is that of a surface $\Sigma$ such that to a point $P$ on the surface corresponds a point $P_{1}$ on one curve and a point $P_{2}$ on the other.*

Let the curves be defined by

$$
\begin{array}{lll}
f\left(x_{1}, x_{2}, x_{3}\right)=0, & x_{1}^{\prime}=0, & x_{2}^{\prime}=0, \\
f^{\prime}\left(x_{1}^{\prime}, x_{2}^{\prime}, x_{3}^{\prime}\right)=0, & x_{1}=0, & x_{2}=0
\end{array}
$$

in the space of four dimensions $S_{4} \equiv\left(x_{1}, x_{2}, x_{3}, x_{1}^{\prime}, x_{2}^{\prime}, x_{3}^{\prime}\right), x_{3}=x_{3}^{\prime}$; and let $\Sigma$ be defined by $f\left(x_{1}, x_{2}, x_{3}\right)=0, f^{\prime}\left(x_{1}^{\prime}, x_{2}^{\prime}, x_{3}^{\prime}\right)=0$. A plane $k_{3} x_{1}=k_{1} x_{3}$, $k_{3} x_{2}=k_{2} x_{3}$. belonging to the conical variety $f=0$ meets $\Sigma$ in the plane curve

* F. Severi, Sulle corrispondenze fra $i$ punti di una rurva algebrica e sopra certe classi di superficie, Memorie della Accademia reałe di Torino, vol. 54 (1903), pp. 1-49. See pp. 19-34. 


$$
f^{\prime}\left(x_{1}^{\prime}, x_{2}^{\prime}, x_{3}^{\prime}\right)=0, \quad k_{3} x_{1}-k_{1} x_{3}=0, \quad k_{3} x_{2}-k_{2} x_{3}=0 .
$$

Thus, $\Sigma$ contains a one-dimensional system of plane curves, each birationally equivalent to the curve (20). Similarly, by intersecting the variety $f=0$ by the planes belonging to $f^{\prime}=0$, we obtain a second system of plane curves, each birationally equivalent to the curve (19). Since two planes in $S_{4}$ meet in one point, it follows that every curve of each system meets every curve of the other in one and only one point.

12. Multiple correspondences on $\Sigma$. Let $K$ be any algebraic curve on $\Sigma$. It meets the curves of one system in $n$ points, and those of the other in $n^{\prime}$ points. Since through every point of $K$ one curve of each system passes, it follows that $K$ establishes an $\left(n, n^{\prime}\right)$ correspondence between the curves (19) and (20). Conversely, any correspondence between these curves may be represented by a curve $K$ on $\Sigma$.

When the correspondence can be defined by one equation, $K$ is a complete intersection on $\Sigma$, and conversely. When the correspondence requires two equations for its definition, $K$ is a partial intersection on $\Sigma$. The results already found by means of ruled surfaces can be readily interpreted in terms of $K$ on $\Sigma$. Thus, when two ruled surfaces have a common generator, their residual intersection corresponds to a curve $K$ on $\Sigma$ which has a double point for each common generator. If two ruled surfaces have a common simple directrix this curve corresponds to the curve of the identical transformation on $\Sigma$ and $\Delta=0$ is a variety through this directrix, meeting $\Sigma$ in a residual curve $K$. Similarly for a multiple directrix.

Moreover, we see that if between two curves exists one multiple correspondence not expressible by means of one equation, then these curves also have other such correspondences formed by passing a variety through the curve of the given correspondence on $\Sigma$, and taking the residual intersection.

13. General criteria. The problem of finding correspondences between two curves that require two equations for their determination may be presented in a different form by commencing with $f(x)=0, \phi\left(x, x^{\prime}\right)=0$, in which the coefficients in each are as yet undetermined. We then find the restrictions on these functions so that sets of points in $(x)$ determined by $f=0, \phi=0$ are rationally separable into two or more sets for values of the $x_{i}^{\prime}$ which satisfy an equation $f^{\prime}\left(x^{\prime}\right)=0$.

Example. Let $f(x)=0, \phi\left(x, x^{\prime}\right)=0$ be respectively

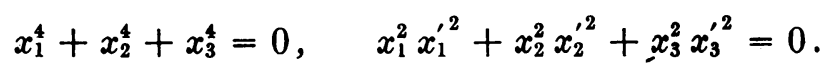

Eliminate $x_{1}$ between these two equations and express the condition that the resultant quadratic in $x_{2}, x_{3}$ is rationally factorable. This requires that the 
expression $x_{1}^{\prime 4}+x_{2}^{\prime 4}+x_{3}^{\prime 4}$ shall be a square for points on $f^{\prime}\left(x^{\prime}\right)=0$. We may put, for example,

Then

$$
f^{\prime}\left(x^{\prime}\right) \equiv\left(x_{1}^{\prime 4}+x_{2}^{\prime 4}+x_{3}^{\prime 4}\right) x_{1}^{\prime 4}=x_{2}^{\prime 6} x_{3}^{\prime 2} \text {. }
$$

$$
\phi_{1}\left(x, x^{\prime}\right)=\left(x_{1}^{\prime 4}+x_{2}^{\prime 4}\right) x_{2}^{2}+x_{2} x_{3} x_{2}^{\prime 2} x_{3}^{\prime 2}+x_{2}^{\prime 3} x_{3}^{\prime} x_{3}^{2}=0 \text {. }
$$

The correspondence between the planes $(x),\left(x^{\prime}\right)$ is $(4,8)$. The genus of $f(x)=0$ is 3 , and of $f^{\prime}\left(x^{\prime}\right)=0$ is 9 .

Cornell Untversity 